\title{
La exposición secundaria al humo de cigarro puede alterar la programación fetal de la presión arterial*
}

$\mathrm{L}$ os bebés nacidos de madres que fuman cigarrillos pueden estar en riesgo de tener controles anormales de la presión arterial y del ritmo cardiaco en el momento de nacer, según sugiere una investigación publicada en el número de marzo de 2010 de la revista Hypertension. Los resultados de este nuevo estudio insinúan, además, que este control puede empeorar si continúa la exposición secundaria al humo del cigarro, tal vez con un mayor riesgo de desarrollar hipertensión en una etapa posterior de la vida.

El estudio comparaba el control del ritmo cardiaco y de la presión arterial de 19 bebés nacidos de madres no fumadoras con los de 17 bebés cuyas madres reportaron fumar un promedio de 15 cigarrillos al día antes y después de dar a luz. La presión arterial en reposo de los bebés de ambos grupos siguió esencialmente la misma tendencia de desarrollo durante el primer año de vida, pero los bebés expuestos al humo presentaron una presión arterial diastólica más elevada a la edad de 3 meses. El ritmo cardiaco en reposo de ambos grupos también fue similar y siguió la misma tendencia hasta la edad de 3 meses. Sin embargo, para el año de edad, el ritmo cardiaco en reposo de los bebés expuestos al humo era en promedio $20 \%$ más lento que el de los bebés no expuestos.

Los investigadores también monitorearon los cambios en el ritmo cardiaco y la presión arterial a lo largo de un lapso de 40 latidos mientras los niños, que dormían profundamente sobre mesas inclinadas eran levantados de una posición supina a una inclinación de $60^{\circ}$ a lo largo de 5 segundos y sostenidos en esa postura durante 1 minuto. "A medida que el cuerpo se yergue más, el ritmo cardiaco debe elevarse temporalmente, y los distintos vasos sanguíneos deben contraerse para incrementar la presión arterial y asegurar que llegue suficiente sangre al cerebro", explica el primer autor Gary Cohen, investigador científico principal del Departamento de Salud Materno Infantil del Instituto Karolinska de Estocolmo. Ciertamente, eso es lo que observaron los autores en los niños no expuestos, alcanzando valores pico un tanto más elevados entre las edades de 1 semana y 1 año, tal como se esperaba.

Los bebés expuestos mostraron una tendencia similar con el tiempo. Sin embargo, entre los 3 meses y el año de edad, sus respuestas se volvieron exageradas, con un ritmo cardiaco más acelerado (con un

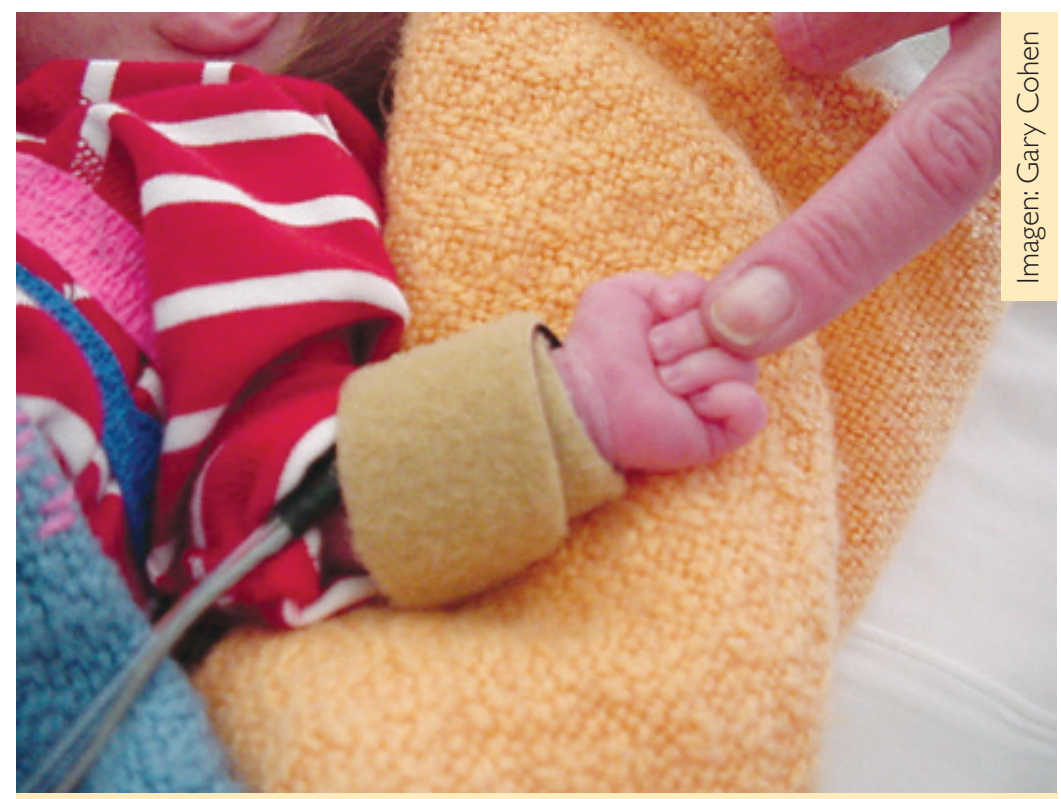

La exposición prenatal al humo del cigarro de la madre puede haber contribuido a las anomalías de la presión arterial observadas en bebés. 
aumento de $11.5 \%$ en promedio, en lugar de $6.5 \%$ observado en los bebés no expuestos) antes de caer más rápidamente. Lo mismo ocurrió con su presión arterial diastólica.

Cuando se inclinó a los bebés y se los mantuvo en posición vertical, se observaron incrementos sostenidos de 2 a 3\% en la presión arterial sistólica, diastólica y media a la semana de edad, y de 8 a 10\% para el año de edad, tal como se esperaba. Por el contrario, en los bebés expuestos, los incrementos en la presión arterial fueron de casi el doble a la semana de edad, pero ya no hubo más incrementos con el paso del tiempo.

"Así, los bebés recién nacidos de madres fumadoras reaccionan de manera excesiva al cambio de postura, pero para cuando llegan a la edad de 1 año y quieren ponerse de pie, reaccionan por debajo de lo normal; sus sistemas ordinarios de compensación de la presión arterial sencillamente no funcionan bien", señala Cohen. "Tal parecería que ni su ritmo cardiaco ni su tono simpático constrictor [impulsos del sistema nervioso simpático que ayudan a controlar la constricción de los vasos sanguíneos] están programados 'adecuadamente' incluso en el momento de nacer, y que las cosas empeoran con el tiempo."

Este problema de programación podría radicar en un tono simpático demasiado fuerte provocado por la exposición a algún compuesto del humo del cigarrillo en la matriz y después del nacimiento, señalan los investigadores. Esto podría incrementar lentamente la resistencia vascular, dando lugar a un incremento en la presión arterial diastólica observada en reposo a la edad de 3 meses, y a

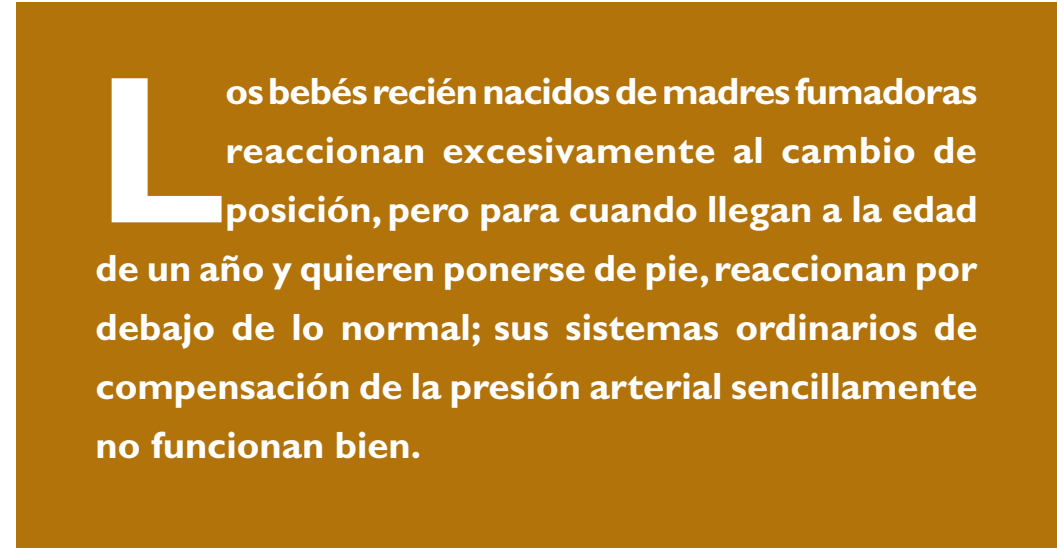

la pérdida eventual de la reactividad simpática.

Los autores plantean además la hipótesis de que una disminución en el ritmo cardiaco observada en los bebés expuestos al año de edad fue un intento de restaurar algún tipo de equilibrio. Por desgracia, esta solución de reprogramación parece obstaculizar el control posicional de la presión sanguínea adecuado, "y hay evidencias de que esto podría incrementar las probabilidades de que se presente hipertensión más adelante", explica Cohen. En los adultos, la patofisiología cardiovascular puede implicar un exceso de actividad simpática crónica que dé lugar a un incremento de la presión sanguínea. Los autores sugieren que puede estar ocurriendo algo similar en los niños que han sido estudiados.

"Se requiere de más investigación para determinar [si estas observaciones pueden] explicarse como una consecuencia de alteraciones en el flujo simpático central, dado que esto no se evaluó directamente en este estudio", comenta James Fisher, profesor de fisiología del ejercicio de la Escuela de Ciencias del Deporte y del Ejercicio de la Universidad de Birmingham que no participó en el estudio. "Como suele suceder con las buenas investigaciones, nos quedan más preguntas que respuestas. La reactividad cardiovascular alterada ¿es específica del estrés postural, o es más generalizada? ¿Cuál es la importancia biológica de la magnitud de la alteración de la reactividad cardiovascular? ¿Qué tan permanente es la 'reprogramación'; ¿ es reversible si se retira la exposición al humo?"

Si bien el estudio es interesante, es bastante pequeño. "Me gustaría ver una confirmación en un estudio más grande", dice Mark Caulfield, director del Instituto de Investigación William Harvey de Barts y de la Escuela de Medicina y Odontología de Londres: "con una prueba formal del nivel de consumo de cigarrillos en cada uno de los grupos estudiados, antes de extraer una conclusión firme."

Adrian Burton es un biólogo que vive en España y escribe periódicamente para las revistas The Lancet Oncology, The Lancet Neurology y Frontiers in Ecology and the Environment. 